\title{
Effective Local Backscattering Estimation for Underwater Image Enhancement
}

\author{
Codruta O. Ancuti ${ }^{\dagger}$, Cosmin Ancuti* and Horia Balta ${ }^{\dagger}$ \\ ${ }^{\dagger}$ MEO, Universitatea Politehnica Timisoara, Romania \\ ${ }^{*}$ Girona University, Institute of Informatics and Applications ,Girona ,Spain
}

\begin{abstract}
Due to absorption and scattering effects the underwater scenes are characterized by poor contrast, color shifting, additional noise and hazy appearance. In this paper we introduce a novel solution that estimates locally the backscattered light. While in general the existing solutions estimate a global backscattered light value over the entire scene, our local strategy is able to deal effectively to the more challenging non-uniform illumination generated by multiple light-sources. Our solution computes two complementary estimates of the local backscattered light, covering a large and a small patch size. The optimal local backscattered light is computed as the mean of the outputs processed with the small and the large patches while the transmission map, is estimated based on the dark-channel prior (DCP) [1]. Finally, our restored results are computed by simply inverting the optical model using the transmission and the local backscattered light estimates. The qualitative evaluation demonstrates the effectiveness of our approach compared with the recent underwater enhancing techniques.
\end{abstract}

Index Terms

underwater, backscattering, dehazing, enhancement

\section{INTRODUCTION}

A various and large number of underwater image-based applications, from control of underwater vehicles to inspection of the underwater infrastructure, archeology or marine biology research, requires reliable image and video enhancement techniques. In underwater scenes the visibility is highly degraded due to the attenuation of the propagated light and due to the absorption and scattering effects. While the absorption effect diminishes the energy of the incident rays, the scattering effect changes the direction of the ray of light. Due to these effects the underwater scenes are characterized by poor contrast, color shifting, additional noise and hazy appearance.

In general the poor visibility of underwater scenes is due to the backscattering. Since traditional enhancing techniques such as gamma correction, histogram equalization have shown strong limitation for underwater image enhancement task, several specialized underwater image enhancing techniques that extend these traditional enhancing techniques for underwater case have been introduced [2], [3], [4]. To deal with extreme backscattering, a first class of techniques applied additional equipments such as polarization filters [5], which in general represents impractical acquisition systems.

More recently, and in general inspired by the recent outdoor image dehazing techniques [10], [11], [12], [13], [14], [15] several underwater enhancing solutions [6], [7], [8], [9], [16], [17], [18], [19] have been introduced in the literature. These techniques reconstruct in general the original scene radiance by inverting the Koschmieder's visibility model [20]. Many of these techniques have been built on the well-known Dark Channel Prior (DCP) [11], [1]. DCP, originally introduced for outdoor dehazing problem, is a statistical prior that assumes that for haze-free outdoor images in a given non-sky patch at least one color channel has intensities very close to zero. Chiang and Chen [21] employs the DCP to segment the foreground and the background regions. Consequently, this segmentation has been used to reduce the haze effects and color variations based on color compensation. Drews-Jr et al. [22] introduce an underwater specific prior directly derived from DCP, called Underwater Dark Channel Prior (UDCP). They assumed that in underwater scenes most of the visual information are contained by the green and blue color channels.

Similarly, Galdran et al. [6] introduced a specific prior called Red channel prior built on the observation that in underwater the intensity of the red channel decreases faster as distance increases. Emberton et al. [7] employ a set of features to find regions that are the most hazeopaque. Then, a hierarchical rank based method is used to estimate the backscattering and the transmission map. Ancuti at al. [19] introduce a fusion-based approach for underwater image enhancement that is shown to perform better than the initial solution [23] in presence of severe light attenuation, while supporting accurate transmission estimation in various acquisition settings. Inspired by the color lines approach of Fattal [24], Lu and al. [25] used DCP to estimate the transmission in underwater. In [18], Ancuti et al. introduces an original strategy that exploits color transfer while tuning the color correction locally, as a function of the light attenuation level estimated from the red channel. This color correction strategy demonstrates also to improve significantly the local keypoints matching for underwater scenes. However, in general the existing single-image underwater enhancing techniques show important limitations for complex situation such as swirling water and/or artificial ambient illumination (see Fig.1).

This work introduces a novel solution that estimates locally the backscattered light. While most of the solutions estimate a global backscattered light value over the entire scene, our local strategy is able to deal effectively to the more challenging non-uniform illumination generated by multiple light-sources that may be present in many underwater scenes (see Fig. 1). Local estimation of the backscattered light has been used recently in the work of Berman et al. [9]. However, we use a different approach inspired by our previous work [16]. In contrast to [16] that uses multi-scale fusion and computes the backscattered light on a grid of patches, here we introduce a simplified approach that is built on the optical model. To circumvent the difficulty of selecting the optimal patch size (accounting for distinct features such as dimensions and colors of objects in the scenes, nature of the ambient light, non-uniform illumination), our strategy employs two complementary estimates of the local backscattered light, covering a large and a small patch size. The large size of the patch aims to recover the global contrast while the small size of the patch deals with the degradation due to the multiple light sources. The optimal local back-scattered light is computed as the mean of the outputs processed with the small and the large patches. The other unknown of the optical model, the transmission map, 
Input image

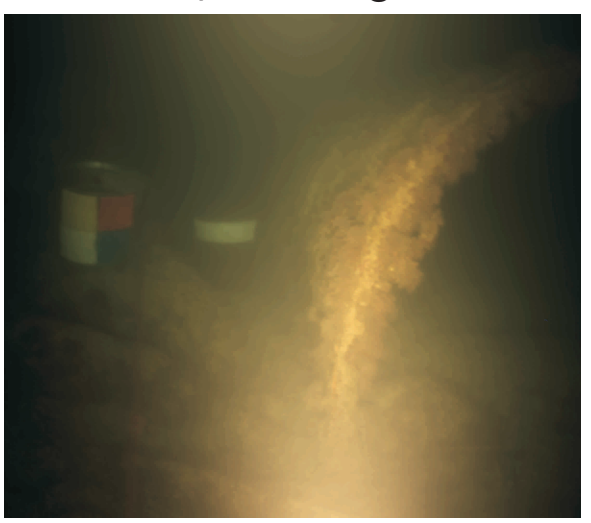

Drew Jr et al.

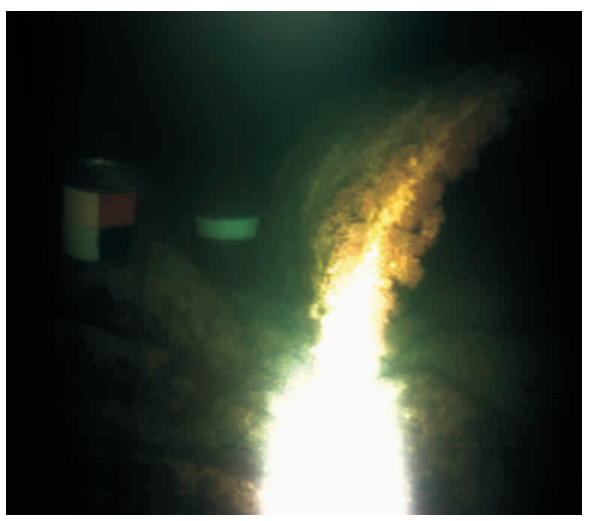

Treibitz and Schechner

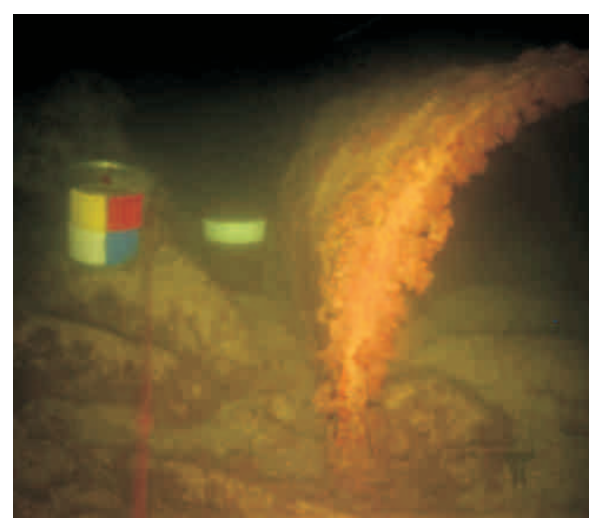

Berman et al.

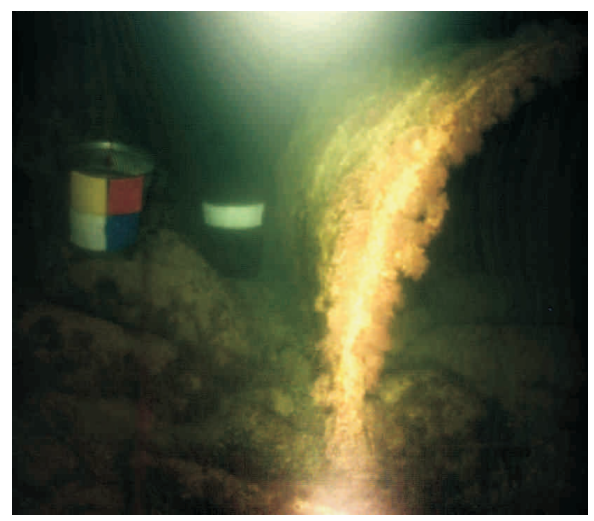

Emberton et al.

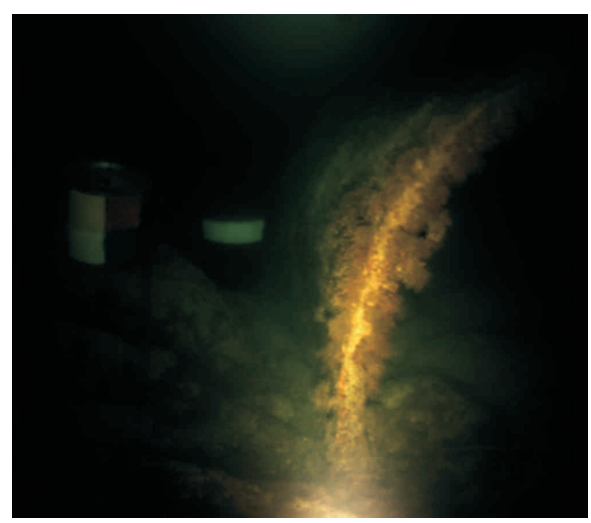

Our result

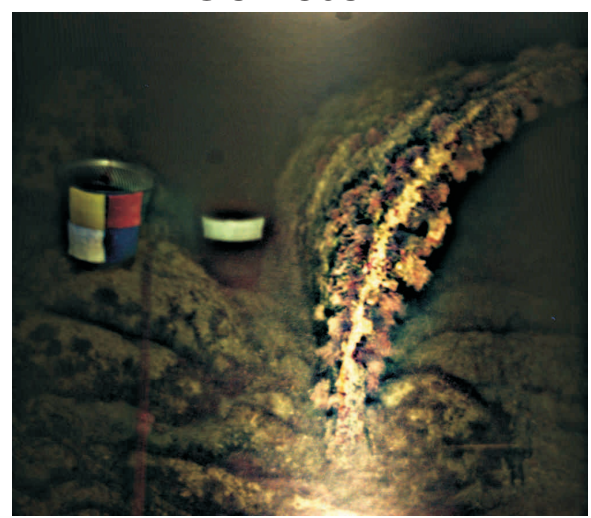

Fig. 1. Specialized underwater restoration methods based on global [6], [7], [8] and local estimation [9] of the backscattered light show important limitations to recover visibility of underwater scenes illuminated by artificial lightning. Our local approach restores both color and contrast (local and global) comparable and even better than polarization-based technique (uses multiple images) of [5].

is estimated based on the dark-channel prior (DCP) [1]. Finally, our restored results are computed by simply inverting the optical model using the transmission and the local backscattered light estimates. The extensive evaluation of our approach demonstrates the effectiveness of our approach compared with the recent underwater enhancing techniques.

\section{UNDERWATER OpticAl MODEL}

In our approach we rely on the well-known optical model of Jaffe-McGlamery [26], that is often employed in the representation of the underwater scenes. Based on this optical model, the total radiance of an image $\mathcal{I}$ that reaches the camera is influenced by three additive components: a direct component $E_{D}$, a forwardscattering component $E_{F S}$ and a backscattering component $E_{B S}$. Considering the fact that underwater medium is not ideal, the energy of a light ray that crosses this medium from a scene object until it reaches the observer is diminished due to the physical phenomena such as absorption and scattering.

Direct component $E_{D}$ is the attenuated signal (dependent by the distance) of the reflected light, and it is estimated at each image coordinate $x$ as:

$$
E_{D}(x)=J(x) e^{-\eta d(x)}=J(x) t(x)
$$

where $J(x)$ represents the radiance of the object, $d(x)$ represents the distance from the observer to the object, and $\eta$ is the attenuation coefficient of the medium. The transmission $t(x)$ is expressed as an exponential term $t(x)=e^{-\eta d(x)}$.

Forward-scattering $E_{F S}$, is the deflection of a portion of the incident light and in general it is neglected since it has a small influence in the overall image degradation process.

Back-scattering or the veiling light [27], expresses both the loss of the contrast and the color shifting of underwater images. Defined in [27], for visually acceptable distances (between 3-10 m) it is expressed as:

$$
E_{B S}(x)=B_{\infty}\left(1-e^{-\eta d(x)}\right)
$$

where $B_{\infty}$ is the back-scattered light or the water background [28] and $d(x)$ is the distance.

The simplified underwater optical model expression considers both direct component and backscattering, but ignores the forwardscattering component and therefore it writes:

$$
\begin{aligned}
\mathcal{I}(x) & =J(x) e^{-\eta d(x)}+B_{\infty}\left(1-e^{-\eta d(x)}\right) \\
& =J(x) t(x)+B_{\infty}(1-t(x))
\end{aligned}
$$


Due to the similarities between the underwater camera model (3) and the optical model of Koschmieder [20] (employed for outdoor image dehazing), recently in the literature have been proposed several methods to restore underwater images that are derived from the outdoor dehazing solutions.

For example, in the work of Chiang and Chen [21] the rough depth map of the underwater scene is estimated based on the Dark Channel Prior (DCP) [1]. Similarly, Galdran et al. [6] computes a variation of DCP named Red Channel Prior that aims to recover colors corresponding to short wavelengths. However, at greater depths when the artificial illumination is required to illuminate the scene, these outdoor/DCP dehazing-inspired techniques perform in general poorly and suffer from important limitations when restoring contrast and color (see Fig. 1).

In the next section we explain how to estimate the two unknowns of the simplified optical model: the transmission term $t(x)$ and the backscattered light value $B_{\infty}$.

\section{LOCAL BACKSCATTERING ESTIMATION}

\section{A. Transmission map estimation}

The generally accepted solutions for the underwater medium apply various interpretations of the Dark Channel Prior (DCP) introduced by He et al. [1] to estimate the transmission $t(x)$ for outdoor hazy scenes. We observe that this solution generates decent results in underwater only if the selective attenuation is not affecting the color channels. To overcome the red channel attenuation (or zero transmission for the affected regions), here we include a simple strategy for the red channel compensation as described recently in [19]. Based on the DCP, the transmission $t(x)$, is computed by finding the smallest value over a neighborhood/patch of $x$ (the dark channel image is referred as $\mathcal{I}_{\mathcal{D C}}(x)$ and is estimated as $\left.\min _{y \in \Omega(x)}\left(\min _{c \in r, g, b} \mathcal{I}^{c}(x)\right)\right)$.

Applying the underwater optical model (3) the transmission is expressed:

$$
t(x)=1-\min _{y \in \Omega(x)}\left(\min _{c \in r, g, b} \mathcal{I}^{c}(x) / B_{\infty}{ }^{c}\right)
$$

where $\Omega(x)$ is a local patch centered at $x$.

In our experiments we observed that $t(x)$ can be reasonably approximated when the backscattered light $B_{\infty}$ is replaced with the (maximum) color intensity vector $[1,1,1]$, so that we have:

$$
t(x) \approx 1-\min _{y \in \Omega(x)}\left(\min _{c \in r, g, b} \mathcal{I}^{c}(x)\right)
$$

\section{B. Backscattering estimation}

The backscattered light $B_{\infty}$ represents the light that is scattered back by floating particles and it is the main factor that generates the loss of contrast and color degradation in underwater images.

In general the existing underwater descattering strategies estimate a global constant value of the backscattered light.

As shown in Fig. 1 and Fig. 3, such global strategies are mislead by specular reflections or glowing effects due to the artificial illumination in the underwater. In contrast, here we consider an alternative solution to the global backscattered light estimation. Inspired by our previous work [16], we first introduces a global estimator expression based on the minimum of $\mathcal{I}^{c}$ over a small neighborhood around $y$, instead of just its (potentially noisy) value in $y$ :

$$
B_{G_{\infty}}^{c}=\max _{y \in M_{D C}^{I}}\left(\min _{z \in \Omega(y)} \mathcal{I}^{c}(z)\right)
$$

where $\Omega(y)$ defines a neighborhood around $y$, and $M_{D C}^{I}$ denotes the set of locations in image $I$ where the dark channel reaches its global maximum value $\mathcal{D} \mathcal{C}_{\text {max }}^{I}$, i.e. $M_{D C}^{I}=\left\{y \mid \mathcal{I}_{\mathcal{D C}}(y)=\mathcal{D C}_{\text {max }}^{I}\right\}$. Hence, instead of keeping an arbitrary 99.9 percentile, we simply keep the whole set of positions that maximize $\mathcal{I}_{\mathcal{D C}}$. Since $\mathcal{I}_{\mathcal{D C}}$ is defined based on a minimization over a patch, $M_{D C}^{I}$ always includes multiple locations. Our extensive validation reveals that this approximation does not impact the global estimation. Moreover, it makes the expression easier to generalize to a local estimator.

Derived from the global estimator expression (Equation 6), we define the backscattered light in location $x$ as:

$$
B_{L_{\infty}}^{c}(x)=\max _{y \in M_{D C}^{\Psi(x)}}\left(\min _{z \in \Omega(y)} \mathcal{I}^{c}(z)\right)
$$

where $\Psi(x)$ is a patch centered in $x$ of $\mathcal{I}_{D C}$. As for the global estimator, $M_{D C}^{\Psi(x)}$ denotes the set of positions in $\Psi(x)$ where the dark channel is maximum, i.e. reaches the value of its local maximum over $\Psi(x)$. In practice, the patch $\Psi$ is typically larger than $\Omega$. We use a default value of 2 for the ratio between the $\Psi$ and $\Omega$ patches. For underwater scenes with relatively uniform illumination and less turbidity, the ratio is recommended to be set to a higher value. An important observation is that the size of $\Omega$ is not trivial to be selected since a large neighborhood means that the result may be influenced by multiple light sources due to inaccurate estimation, which results in color shifting. In contrast, a smaller neighborhood reduces the effectiveness of the dark channel prior and consequently the effectiveness of the haze removal.

To mediate this complementary cases, we compute two different estimates of the local $B_{L_{\infty}}^{c}(x)$. The first one is generated for a relatively large patch size (e.g. 15\% of the image size) and aims to improve the image contrast by reducing the haze effect, with the risk of introducing color shifting. The second one is generated computing the estimate for a smaller patch size (e.g. 3\% of the image size). Although this might underestimate the backscattered light value, this shows to be effective to remove the glowing effect. To compute our optimal local backscattering estimate, we simply average all the local values obtained when computing the two different size patches. Finally, to generate our results we simply invert the optical model using the optimal estimate of backscattering and transmission as previously described. 

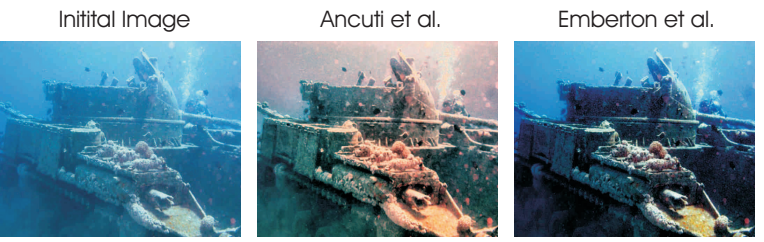

Galdran et al.

Drews-Jr et al.
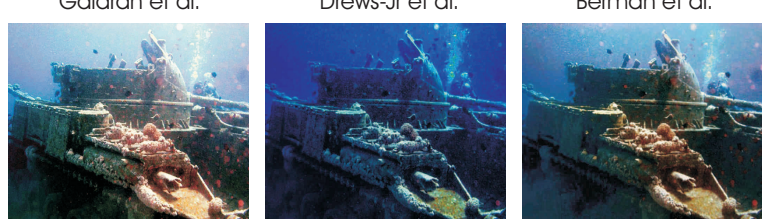

Our Results
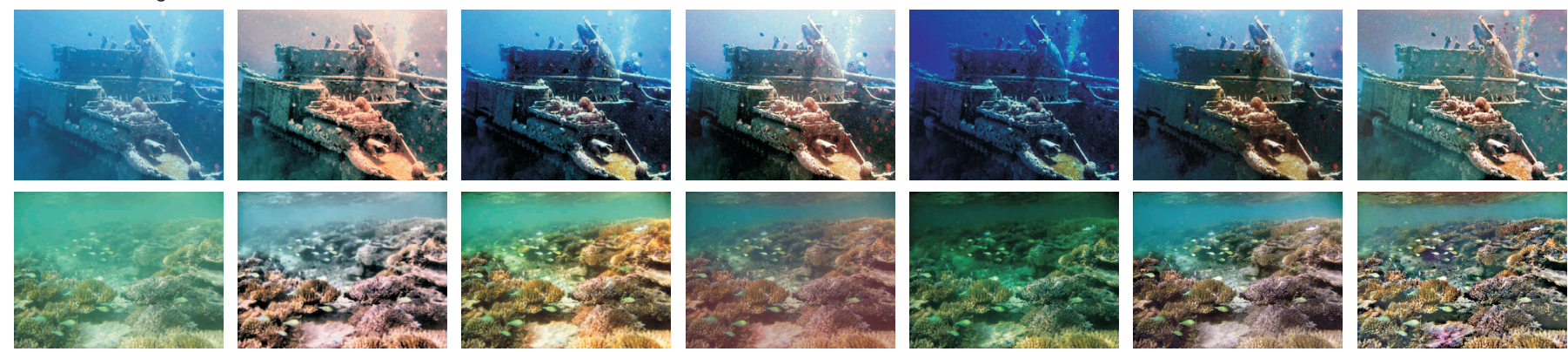

Fig. 2. Comparative results of the techniques [23], [7], [6], [8], [9] for underwater scenes with uniform lightning.
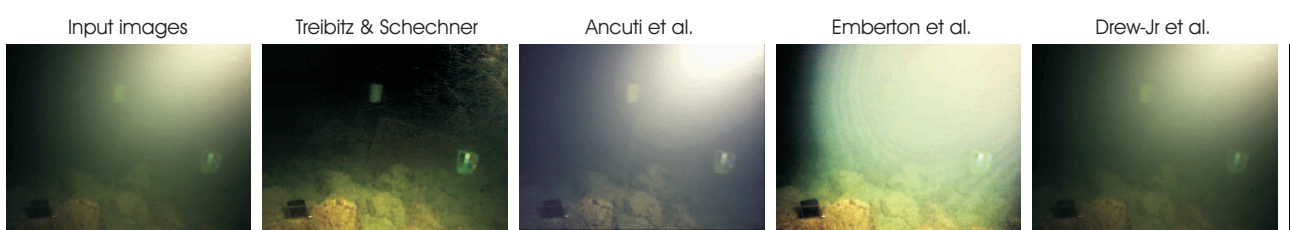

Berman et al
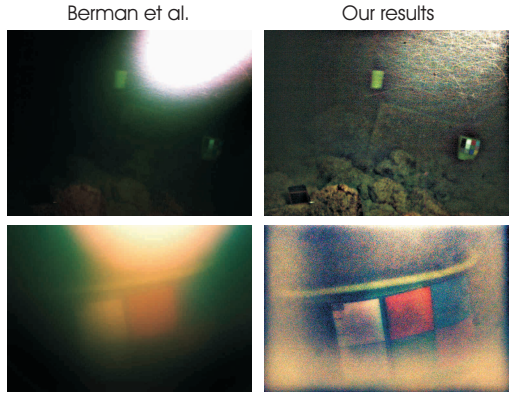

Fig. 3. Comparative results of the techniques [5], [23], [7], [8], [9] for underwater scenes with non-uniform lightning. Our method restores the visibility comparable and even better than polarization-based technique (that uses multiple images) of [5] and the approach of Berman et al. [9] that also estimates locally the backscattering.

\section{RESUlTS AND Discussion}

We extensively tested our approach for a large dataset of underwater images. In our experiments we considered several recent underwater enhancing techniques including the techniques of Ancuti et al. [23], Emberton et al [7], Galdran et al [6], Drews-Jr et al.[8] and Berman et al.[9].

In Fig. 2 are shown two underwater images of scenes with relatively uniform illumination. As can be seen our method yields comparative and even better results when compared with the evaluated underwater techniques. On the other hand in Fig. 1 and Fig.3 are shown images of non-uniform (artificially) illuminated underwater scenes. As can be observed our solution is very competitive also for such challenging cases even compared with the local approach of Berman et al.[9].

To conclude, in this paper we present an original solution that estimates locally the backscattered light in underwater scenes. We compute two complementary estimates of the local backscattered light, covering a large and a small patch size. The optimal local backscattered light is computed as the mean of the outputs processed with the small and the large patches while the transmission map, is estimated based on the dark-channel prior (DCP) [1]. The qualitative evaluation demonstrates the effectiveness of our approach compared with the recent underwater enhancing techniques.

\section{ACKNOWLEDGMENT}

Part of this work has been funded by the Romanian Government UEFISCDI, project PN-III-P2-2.1-PED-2016-0940. Part of this work has been funded from the 2020 European Union Research and Innovation Horizon 2020 under the grant agreement Marie Sklodowska-Curie No 712949 (TECNIOspring PLUS), as well as the Agency for the Competitiveness of the Company of the Generalitat de Catalunya - ACCIO: TECSPR17-1-0054. 


\section{REFERENCES}

[1] K. He, J. Sun, and X. Tang, "Single image haze removal using dark channel prior," IEEE TPAMI, 2011.

[2] S. Bazeille, L. Jaulin I. Quidu, and J. P. Malkasse, "Automatic underwater image pre-processing," In Proc. of CMM, 2006.

[3] A. Arnold-Bos, J.-P. Malkasset, and G. Kervern, "Towards a model-free denoising of underwater optical images," IEEE Europe Oceans Conf., 2005.

[4] F. Petit, A.-S. Capelle-Laize, and P. Carre , "Underwater image enhancement by attenuation inversion with quaternions," IEEE ICASSP, 2009.

[5] T. Treibitz and Y.Y. Schechner, "Active polarization descattering," IEEE Trans Pattern Anal Mach Intell., 2009.

[6] A. Galdran, D. Pardo, A. Picon, and A. Alvarez-Gila, "Automatic red-channel underwater image restoration," Journal of Visual Communication and Image Representation, 2015.

[7] S. Emberton, L. Chittka, and A. Cavallaro, "Hierarchical rank-based veiling light estimation for underwater dehazing," Proc. of British Machine Vision Conference (BMVC), 2015.

[8] P.L.J. Drews Jr., E. R. Nascimento, S.S.C. Botelho, and M. F. M. Campos, "Underwater depth estimation and image restoration based on single images," IEEE CGA, 2016

[9] T. Treibitz D. Berman and S. Avidan, "Diving into haze-lines: Color restoration of underwater images," Proc. of British Machine Vision Conference (BMVC), 2017.

[10] R. Fattal, "Single image dehazing," SIGGRAPH, 2008.

[11] K. He, J. Sun, and X. Tang, "Single image haze removal using dark channel prior," In IEEE CVPR, 2009.

[12] J.-P. Tarel and N. Hautiere, "Fast visibility restoration from a single color or gray level image," In IEEE ICCV, 2009.

[13] J.-P. Tarel, N. Hautiere, L. Caraffa, A. Cord, H. Halmaoui, and D. Gruyer, "Vision enhancement in homogeneous and heterogeneous fog," IEEE Intelligent Transportation Systems Magazine, vol. 4, no. 2, pp. 6-20, April 2012.

[14] L. Kratz and K. Nishino, "Factorizing scene albedo and depth from a single foggy image," ICCV, 2009.

[15] C.O. Ancuti and C. Ancuti, "Single image dehazing by multi-scale fusion," IEEE Transactions on Image Processing, vol. 22(8), pp. 3271-3282, 2013.

[16] C. Ancuti, C. O. Ancuti, C. De Vleeschouwer, R. Garcia, and A. C. Bovik, "Multi-scale underwater descattering," Int. Conf. on Pattern Recognition (ICPR), 2016.

[17] C. O. Ancuti, C. Ancuti, C. De Vleeschouwer, L. Neumann, and R. Garcia, "Color transfer for underwater dehazing and depth estimation," IEEE Int. Conf. on Image Processing (ICIP), 2017.

[18] C. O. Ancuti, C. Ancuti, C. De Vleeschouwer, and R. Garcia, "Locally adaptive color correction for underwater image dehazing and matching," IEEE CVPR, NTIRE workshop, 2017

[19] C. O. Ancuti, C. Ancuti, C. De Vleeschouwer, and P. Bekaert, "Color balance and fusion for underwater image enhancement," in IEEE Transactions on Image Processing, 2018.

[20] H. Koschmieder, "Theorie der horizontalen sichtweite," in Beitrage zur Physik der freien Atmosphare, 1924.

[21] J. Y. Chiang and Y. Chen, "Underwater image enhancement by wavelength compensation and dehazing," In IEEE Trans. on Image Processing, 2012.

[22] P. Drews-Jr, E. Nascimento, F. Moraes, S. Botelho, M. Campos, and R. Grande-Brazil, "Transmission estimation in underwater single images," IEEE Workshop ICCV, 2013.

[23] C. Ancuti, C. O. Ancuti, T. Haber, and P. Bekaert, "Enhancing underwater images and videos by fusion," in IEEE Conference on Computer Vision and Pattern Recognition (CVPR), 2012.

[24] R. Fattal, "Dehazing using color-lines," ACM Trans. on Graph., 2014.

[25] H. Lu, Y. Li, L. Zhang, and S. Serikawa, "Contrast enhancement for images in turbid water," Journal of the Optical Society of America A, vol. 32, no. 5, pp. 886-893, May 2015.

[26] B. L. McGlamery, "A computer model for underwater camera systems," Ocean Optics, 1979.

[27] Y. Y. Schechner and N. Karpel, "Recovery of underwater visibility and structure by polarization analysis," IEEE Journal of Oceanic Engineering, 2005.

[28] J. N. Lythgoe and C. C. Hemmings, "Polarized light and underwater vision," Nature, vol. 213, no. 79, 1967. 